\title{
ON THE PLANE TERM RANK OF A THREE DIMENSIONAL MATRIX
}

\author{
R. A. BRUALDI ${ }^{1}$ AND J. CSIMA ${ }^{2}$
}

\begin{abstract}
A lower bound is obtained for the plane term rank of a three dimensional matrix in which each of the horizontal two dimensional matrices has constant row and column sums.
\end{abstract}

1. Introduction. Let $A=\left[a_{i j k}\right], 1 \leqslant i, j, k \leqslant n$, be a three dimensional matrix of 0's and l's of order $n$. A plane (respectively, line) of $A$ is defined to be the two dimensional matrix (respectively, one dimensional matrix or vector) which results when one (respectively, two) of $i, j, k$ is held fixed. We distinguish three types of planes: the row planes ( $i$ fixed), the column planes $(j$ fixed) and the horizontal planes ( $k$ fixed). Each line of $A$ is a line (that is, row or column) of two planes of $A$. The plane term rank of $A, \rho(A)$, is $r$ if $r$ is the largest number such that there exist $r$ entries of $A$ which equal 1 with no two of the entries in the same plane of $A$. The plane term rank of $A$ is one of the two possible generalizations of the term rank of a two dimensional matrix of 0 's and l's. It is well known that the analogue for the plane term rank of König's theorem concerning the equality of the term rank and covering number of a two dimensional matrix is false. It is thus of some interest to obtain estimates for the plane term rank.

In [1] we proved that if $A$ is a three dimensional matrix of 0 's and 1's of order $n$ with exactly $c$ l's in each line, then the plane term rank satisfies $\rho(A) \geqslant \min \{n / 2, c\}$.

Koksma [2] has proved that a latin square of order $n$ has a partial transversal of length at least $(2 / 3) n+1 / 3$. A latin square $L$ of order $n$ can be regarded as a three dimensional matrix $A(L)=\left[a_{i j k}\right]$ of 0 's and 1's of order $n$ whereby $a_{i j k}=1$ if and only if the $(i, j)$-entry of $L$ is $k$. Such a matrix has exactly one 1 in each line; conversely, every matrix of this type corresponds to a latin square of order $n$. A partial transversal of length $r$ of the latin square $L$ corresponds to a set of $r$ l's of the matrix $A(L)$ with no two of the l's in the same plane. Thus Koksma's result can be restated as follows: If $A$ is a three dimensional matrix of 0 's and l's of order $n$ with exactly one 1 in each line, then $\rho(A) \geqslant(2 / 3) n+1 / 3$. In this note we obtain a bound for the plane term rank of three dimensional matrices in which each of the horizontal planes has constant row and column sums (which may vary with the horizontal plane).

2. Results. For a real number $a$ we set $p(a)=[a]$ if $a$ is not an integer and $p(a)=a-1$ if $a$ is an integer.

Received by the editors November 14, 1974.

AMS (MOS) subject classifications (1970). Primary 05B20; Secondary 05A05.

${ }^{1}$ Research supported in part by the National Science Foundation Grant No. GP-37978X.

2 Research supported in part by the National Research Council of Canada Grant No. A4078. 
THEOREM 1. Let $A$ be a three dimensional matrix of 0's and 1's of order $n$ with the following properties:

(1) For each $k=1, \ldots, n$ the $k$ th horizontal plane has exactly $c_{k} 1$ 's in each row and column.

(2) For an integer $c$ with $c_{k} \geqslant c(k=1, \ldots, n)$, each of the row planes of $A$ has no $n-1-p((c /(c+1)) n)$ by $2 n-2 p((c /(c+1)) n)$ submatrix of all 0 's.

Then $\rho(A) \geqslant(c /(c+1)) n$.

Proof. Let $\rho(A)=t$. Without loss of generality, we may assume that $a_{k k k}=1$ for $k=1, \ldots, t$. For each $k=1, \ldots, n$ we consider the $k$ th horizontal plane $H_{k}$ to be partitioned into submatrices $H_{k, 1}, H_{k, 2}, H_{k, 3}, H_{k, 4}$ as indicated in the figure below. Since $\rho(A)=t, H_{k, 4}$ is a matrix of all 0 's for $k=t+1, \ldots, n$.

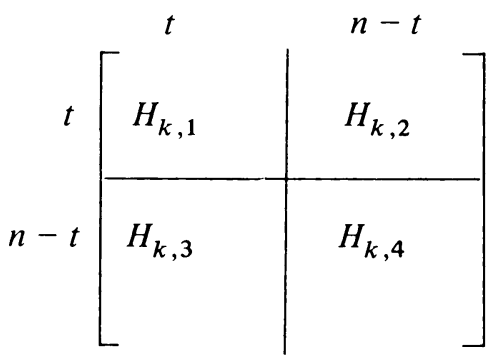

Consider the horizontal plane $H_{t+1}$. Suppose no two l's in $H_{t+1,3}$ were in the same column. Since there are $c_{t+1}(n-t)$ l's in $H_{t+1,3}$, this would imply that $t \geqslant c_{t+1}(n-t) \geqslant c(n-t)$, so that $t \geqslant(c /(c+1)) n$. Thus we may suppose that for some $j_{0}$ with $1 \leqslant j_{0} \leqslant t$, column $j_{0}$ of $H_{t+1,3}$ contains a 1 in the $p$ th row and $q$ th row where $1 \leqslant p<q \leqslant n-t$.

Suppose there were a 1 in $H_{j_{0}, 4}$. This 1 cannot be in both the $p$ th row and $q$ th row of $H_{j_{0}, 4}$. Without loss of generality, we may assume it is in row $u \neq p$; let this 1 be in the column $v$ of $H_{j_{0}, 4}$. Then each of the positions $(1,1,1), \ldots$, $\left(j_{0}-1, j_{0}-1, j_{0}-1\right),\left(j_{0}+1, j_{0}+1, j_{0}+1\right), \ldots,(t, t, t),\left(t+p, j_{0}, t+1\right),(t$ $\left.+u, t+v, j_{0}\right)$ of $A$ is occupied by a 1 , and this implies that $\rho(A) \geqslant t+1$. This is a contradiction, so that we conclude that $H_{j_{0}, 4}$ is a matrix of 0 's. Thus the $c_{j_{0}}(n-t)$ l's in columns $t+1, \ldots, n$ of $H_{j_{0}}$ occur in $H_{j_{0}, 2}$. Since there are $c_{j_{0}}$ 1 's in each row of $H_{j_{0}}$, this implies that $H_{j_{0}, 2}$ has 1 's in at least $n-t$ different rows.

In addition, row $j_{0}$ of $H_{j_{0}, 2}, H_{t+2,2}, \ldots, H_{n, 2}$ must consist of all 0 's. For if there were a 1 in row $j_{0}$ of any of these, then this 1 , the specified 1 in row $p$ of $H_{t+1,3}$ and the 1 's in positions $(1,1,1), \ldots,\left(j_{0}-1, j_{0}-1, j_{0}-1\right),\left(j_{0}+1, j_{0}\right.$ $\left.+1, j_{0}+1\right), \ldots,(t, t, t)$ would imply that $\rho(A) \geqslant t+1$, a contradiction.

Let rows $i_{1}, \ldots, i_{n-t}$ of $H_{j_{0}, 2}$ each contain at least one 1 where $1 \leqslant i_{1}<\ldots$ $<i_{n-t} \leqslant t$ and $i_{k} \neq j_{0}$ for $k=1, \ldots, n-t$. Consider an $i_{r}$. If the $\left(j_{0}, i_{r}\right)$-entry of any of $H_{t+2}, \ldots, H_{n}$ were a 1 , then a 1 in row $i_{r}$ of $H_{j_{0}, 2}$, the 1 in the $\left(j_{0}, i_{r}\right)$ position of one of $H_{t+2}, \ldots, H_{n}$, the specified 1 in row $p$ of $H_{t+1.3}$ along with the l's in the $t-2$ positions of the set

$$
\{(1,1,1), \ldots,(t, t, t)\} \backslash\left\{\left(j_{0}, j_{0}, j_{0}\right),\left(i_{r}, i_{r}, i_{r}\right)\right\}
$$

lead to the conclusion again that $\rho(A) \geqslant t+1$. Thus the $\left(j_{0}, i_{r}\right)$-entry of each of $H_{t+2}, \ldots, H_{n}$ is 0 . 
We conclude that the submatrix of the $j_{0}$ th row plane $R_{j}$ of $A$ which lies at the intersection of rows $t+2, \ldots, n$ and columns $i_{1}, \ldots, i_{n-t}, t+1, \ldots, n$ consists of all 0's. Thus $R_{j}$ has an $n-t-1$ by $2(n-t)$ submatrix of all 0 's.

Now suppose that $t<(c /(c+1)) n$, so that $t \leqslant p((c /(c+1)) n)$. Then

$$
\begin{aligned}
& n-t-1 \geqslant n-1-p\left(\left(\frac{c}{c+1}\right) n\right) \text { and } \\
& 2(n-t) \geqslant 2 n-2 p\left(\left(\frac{c}{c+1}\right) n\right),
\end{aligned}
$$

and we have contradicted (2). We conclude that $t \geqslant(c /(c+1)) n$, and the theorem is proved.

Recall that an $n \times n$ matrix of 0's and 1's is called fully indecomposable if it has no $r \times s$ submatrix of 0 's with $r+s=n(r, s \geqslant 1)$.

TheOREM 2. Let $A$ be a three dimensional matrix of 0 's and 1 's of order $n$ satisfying:

(3) For each $k=1, \ldots, n$ the kth horizontal plane has $c_{k}(\geqslant 2) 1$ 's in each row and column.

(4) Each of the row planes of $A$ is a fully indecomposable matrix. Then $\rho(A) \geqslant(2 / 3) n$.

Proof. By taking $c=2$ in Theorem 1 it is enough to show that the hypotheses imply that each of the row planes of $A$ has no $n-1-p((2 / 3) n)$ by $2 n-2 p((2 / 3) n)$ submatrix of all 0 's. But

$$
n-1-p\left(\left(\frac{2}{3}\right) n\right)+2 n-2 p\left(\left(\frac{2}{3}\right) n\right)=3 n-1-3 p\left(\left(\frac{2}{3}\right) n\right)
$$

which equals $n+2, n+1$, or $n$ according as whether $n$ is congruent to 0,1 , or 2 modulo 3. Since each row plane of $A$ is assumed to be fully indecomposable, the theorem follows from Theorem 1.

\section{REFERENCES}

1. R. A. Brualdi and J. Csima, Extremal plane stochastic matrices of dimension three, Linear Algebra and Appl. 11 (1975), 105-133.

2. K. K. Koksma, A lower bound for the order of a partial transversal in a Latin square, J. Combinatorial Theory 7 (1969), 94 -95. MR 39 \#1342.

Department of Mathematics, University of Wisconsin, Madison, Wisconsin 53706

Department of Mathematics, McMaster University, Hamilton, Ontario, Canada L8S $4 \mathrm{~K} 1$ 